\title{
Recent results from ATHENA
}

\author{
G. Bonomi ${ }^{a *}$ M. Amoretti ${ }^{b}$, P. D. Bowe ${ }^{c}$, C. Canali ${ }^{b d}$, \\ C. Carraro $^{b d}$, C. L. Cesar ${ }^{e}$, M. Charlton ${ }^{f}$, M. Doser $^{a}$,
} A. Fontana ${ }^{g h}$, M. C. Fujiwara ${ }^{i l}$, R. Funakoshi, P. Genova ${ }^{g h}$, J. S. Hangst ${ }^{c}$, R. S. Hayano ${ }^{l}$, L. V. Jørgensen ${ }^{f}$, A. Kellerbauer ${ }^{a}$, V. Lagomarsino ${ }^{b d}$, R. Landua ${ }^{a}$, E. Lodi Rizzini ${ }^{h m}$ M. Macrí ${ }^{b}$, N. Madsen ${ }^{c}$, G. Manuzio ${ }^{b d}$, P. Montagna ${ }^{g h}$, D. Mitchard ${ }^{f}$, A. Rotondi ${ }^{g h}$, G. Testera ${ }^{b d}$, A. Variola ${ }^{b}$, L. Venturelli ${ }^{h m}$, Y. Yamazaki ${ }^{i}$, D. P. van der Werff, N. Zurlo ${ }^{h m}$ ${ }^{a}$ PH Department, CERN, 1211 Geneva 23, Switzerland

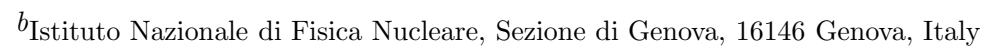
${ }^{c}$ Department of Physics and Astronomy, University of Aarhus, 8000 Aarhus C, Denmark ${ }^{d}$ Dipartimento di Fisica, Università di Genova, 16146 Genova, Italy ${ }^{e}$ Instituto de Fisica, Universidade Federal do Rio de Janeiro, Rio de Janeiro 21945-970, Brazil

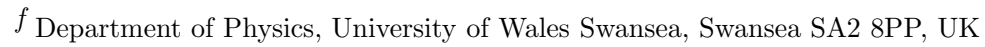
$g$ Dipartimento di Fisica Nucleare e Teorica, Università di Pavia, 27100 Pavia, Italy

$h$ Istituto Nazionale di Fisica Nucleare, Sezione di Pavia, 27100 Pavia, Italy

${ }^{i}$ Atomic Physics Laboratory, RIKEN, Saitama 351-0198, Japan

${ }^{l}$ Department of Physics, University of Tokyo, Tokyo 113-0033, Japan

${ }^{m}$ Dipartimento di Chimica e Fisica per l'Ingegneria e per i Materiali, Università di Brescia, 25123 Brescia, Italy

${ }^{*}$ Now at: Dipartimento di Meccanica, Facoltà di Ingegneria, Università di Brescia - 25123 Brescia, Italy 


\begin{abstract}
The ATHENA experiment at CERN was dedicated to the production of cold antihydrogen atoms by mixing of antiprotons and a positron plasma. The first production took place in 2002 [M. Amoretti et al., Nature 419 (2002) 456]. Results of studies of the production mechanisms of antihydrogen will be described.
\end{abstract}

\title{
1 Introduction
}

The main physics goal of obtaining a sample of trapped and cold antihydrogen $(\bar{H})$ atoms is to study, with spectroscopic methods, their atomic structure and to compare it with that of hydrogen. In this way a direct test of CPT invariance may be performed. The precision of such measurements depends on many parameters, but a relative precision of $10^{-18}$ could, in principle, be achieved. Antihydrogen is a mostly electromagnetic system (weak interaction, or parity violating, effects are small and the same for hydrogen and antihydrogen) thus the comparison between matter and antimatter system is less model dependent.

Another possible experiment utilising a neutral antimatter bound state sample is the measurement of the antimatter gravitational acceleration on earth in order to test the Weak Equivalence Principle (WEP). This states that the gravitational acceleration of a falling object is independent of its composition, such that antimatter should fall on Earth with the same acceleration as matter. No direct measurements exist for the gravitational force on antimatter, therefore even a measurement performed with relatively low precision will represent an important milestone. Antihydrogen is a neutral system thus free from problems associated with electromagnetic interactions that made gravity measurements so difficult with charged antiparticles.

Progress with antihydrogen towards precise CPT and WEP tests started in 1996 when the PS210 experiment at CERN reported the production of the first 9 atoms of antihydrogen [1]. Soon after the E862 experiment at Fermilab confirmed, with another 100 antiatoms, that the creation of antihydrogen was possible [2]. Both of these (storage ring) experiments generated in-flight antiatoms with a very low efficiency and at high energies, rendering difficult any further experimentation. The next generation experiments, ATHENA and ATRAP at CERN, were designed to efficiently produce cold antihydrogen inside an electromagnetic trap. In 2002 first ATHENA [3] and then ATRAP [4] reported the creation of samples of cold antihydrogen by mixing antiprotons $(\bar{p} \mathrm{~s})$ and positrons $\left(e^{+} \mathrm{s}\right)$ at low temperature in a nested Penning trap [5].

\section{Experimental setup}

To produce antihydrogen antiprotons and positrons are obviously needed. The ATHENA apparatus [6] (see Fig. 1) uses antiprotons delivered by CERN's Antiproton Decelerator $(\mathrm{AD})[7]$ and positrons emitted from a ${ }^{22} \mathrm{Na}$ radioactive source $\left(1.4 \times 10^{9} \mathrm{~Bq}\right)$. Both the $\bar{p} \mathrm{~s}$ and the $e^{+} \mathrm{s}$ are trapped, cooled and accumulated in their specific traps prior to moving and mixing in a common trap (called the mixing trap) in the central region of a $3-\mathrm{T}$ 


\section{Capture/mixing trap}

Positron accumulator

${ }^{22} \mathrm{Na}$ source

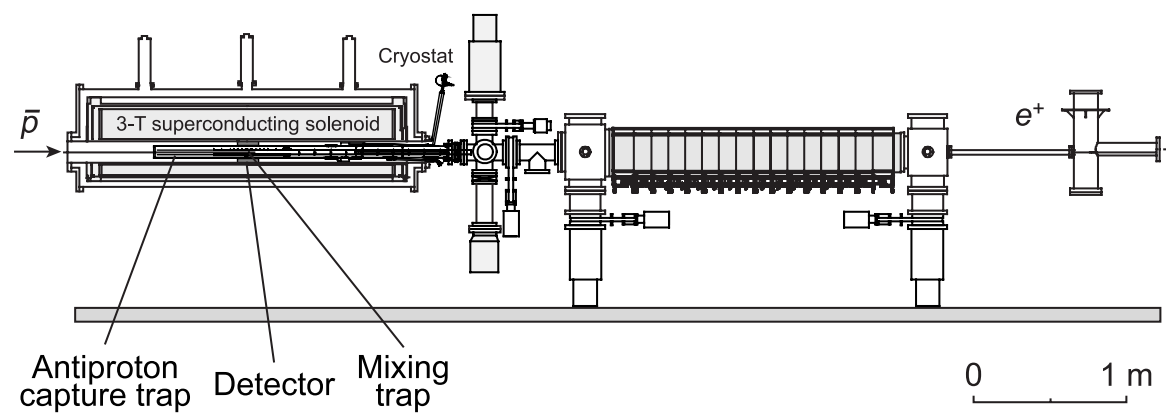

Figure 1: Overview of the ATHENA apparatus. Shown on the left is the superconducting 3-T solenoid magnet which houses the capture trap, the mixing trap, and the antihydrogen annihilation detector. On the right, the radioactive sodium source for positron production and the 0.14-T accumulation trap are located.

superconducting solenoid.

The positron accumulation trap is located inside a room temperature vacuum chamber in a $0.14 \mathrm{~T}$ magnetic field. The antiproton capture trap and the mixing trap are located in the 3-T field of a superconducting magnet (see Fig. 1) whose bore is kept at liquid-nitrogen temperature. A liquid-helium cryostat, whose cold nose protrudes into the magnet bore and encloses the trap, reduces the temperature of the trap region to about $15 \mathrm{~K}$. Ultrahigh vacuum conditions $\left(<10^{-12}\right.$ mbar $)$ are also provided. The 3 -T solenoidal magnetic field which provides the radial confinement also allows positrons to cool efficiently, with a time constant of about $0.5 \mathrm{sec}$, to the trap temperature by the emission of synchrotron radiation [8].

In a "standard mixing cycle" the mixing trap is configured as nested Penning trap [5] (see Fig. 2a), a configuration that allows simultaneous trapping of oppositely charged particles. The central part of the trap is then filled with about 3-7 $\times 10^{7} e^{+} \mathrm{s}$. Once the positrons have self-cooled, about $10^{4} \bar{p}$ s are injected and the two particle species allowed to interact for about 1-3 minutes. At the start of each mixing cycle the antiprotons pass through the positrons many times and are cooled; after few tens of ms antihydrogen formation begins $[9,10]$. At the end of the mixing cycle the nested trap is emptied and both the number of positrons and the number of antiprotons are counted before the process is restarted.

An imaging particle detector [11], used for identification of the $\bar{H}$ annihilation products, surrounds the mixing trap (see Fig. $2 \mathrm{~b}$ ). When formed inside the mixing trap, neutral $\bar{H}$ atoms that survive collisions and field ionization, escape the confinement region and annihilate on the trap electrodes producing a signal in the surrounding vertex detector that triggers the detector readout with an efficiency of $85 \pm 10 \%$. The byproducts of the annihilations (charged $\pi \mathrm{s}$ from the $\bar{p}, \gamma_{\mathrm{s}}$ from the $e^{+}$) are then reconstructed. 

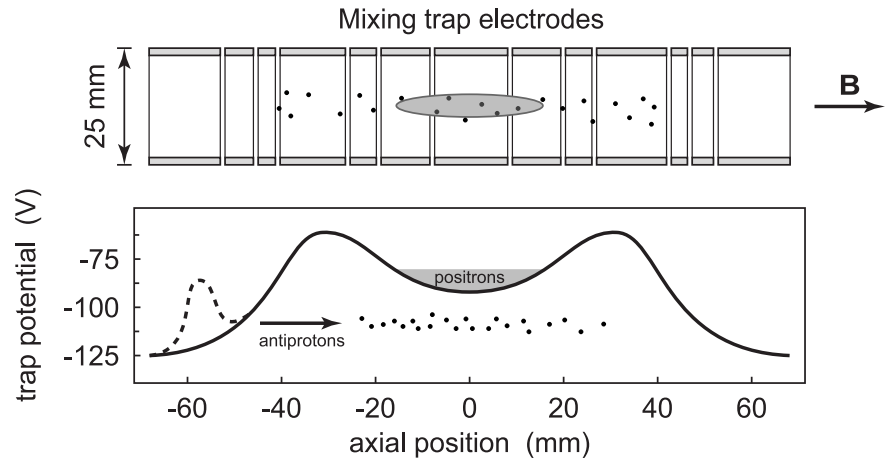

(a)
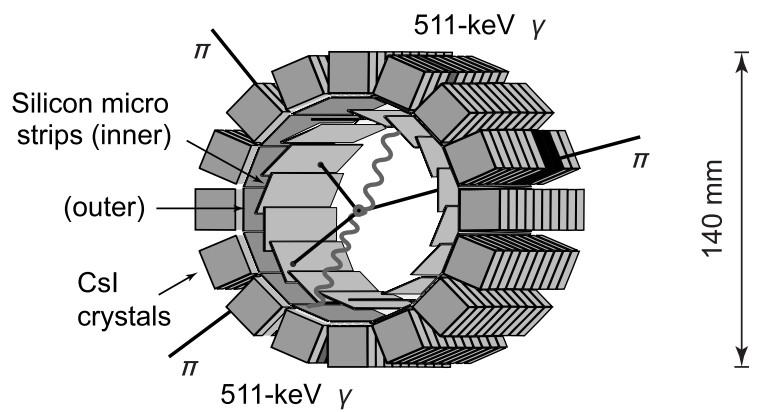

(b)

Figure 2: (a) Detailed sketch of the mixing trap, which is operated in a nested-trap configuration. The graph shows the axial trap potential before (dashed line) and after (solid line) the antiproton injection. (b) Sketch of the antihydrogen annihilation detector. With its highly granular silicon strip and CsI crystal modules, it allows a direct and unambigous detection of $\bar{H}$ production.

\section{Antihydrogen production}

The first production of antihydrogen was reported in 2002 [3]. Fig. 3 reports the signal of such production. The two top plots show the azimuthal distribution of the $\bar{p}$ annihilation vertices. The left diagram is for "normal" mixing conditions (cold mixing), while for the right one the positron plasma is heated up to $3000 \mathrm{~K}$, and antihydrogen production inhibited $([10,12,13])$. In the first case annihilations took place on the trap electrodes because $\bar{H}$, being neutral, left the trap nearly isotropically and struck the trap walls (the electrode ring is clearly imaged in this plot); in the second case the antiprotons could only annihilate in the center on rest gas or ions. The two bottom plots show the distribution of the cosine of the opening angle $\theta_{\gamma \gamma}$ of the two $511-\mathrm{keV} \gamma$ rays recorded in time coincidence with the charged-particle hits, as seen from the charged-particle vertex. The clear excess 
at $\cos \left(\theta_{\gamma \gamma}\right)=-1$ (corresponding to a back-to-back emission of the two $\gamma_{\mathrm{s}}$ typical of the $e^{+}-e^{-}$annihilation) is a proof of the presence of antihydrogen. The right bottom plot distribution corresponds to pure $\bar{H}$ annihilations (Monte Carlo sample). Note that the flat part of the distribution is also due to antihydrogen signal, in cases for which the detector, or the reconstruction software, were inefficient in the detection of both $\gamma \mathrm{s}$. The major detection inefficiency arises from the small volume of the CsI crystals (about $1 \mathrm{~cm}^{3}$ ), that are able to register a $511-\mathrm{keV} \gamma$ only $20 \%$ of the times. Further details of the antihydrogen signal selection and detection have been given elsewhere $[3,10,14]$. It is important to recall here that $65 \pm 5 \%$ in 2002 and $70 \pm 5 \%$ in 2003 of the triggers generated in the ATHENA detector were due to antihydrogen annihilations. The remainder was caused by annihilations of antiprotons on residual gas or ions in the center of the trap, this being lower in better vacuum conditions. The ATHENA collaboration produced a total of more than 2 millions antihydrogen atoms during data taking from 2002 to 2004.

\section{Results}

\subsection{Studies of antihydrogen production parameters}

In the ATHENA experimental conditions two main processes are expected to result in $\bar{H}$ formation: radiative $\left(e^{+}+\bar{p} \rightarrow \bar{H}+\gamma\right)$ and three-body combination $\left(e^{+}+e^{+}+\bar{p} \rightarrow \bar{H}+e^{+}\right)$. In both cases the excess energy is carried away by a third body, being a photon in the first process and a positron in the second one. Extensive discussions of these two processes can be found in $[15,16,17]$ and references therein. They are expected to have the following dependence on the positron plasma density and temperature $\left(n\right.$ and $T^{-0.63}$ for the radiative [17], $n^{2}$ and $T^{-9 / 2}$ [18] for the three-body). Also the expected rates are very different: few tens $s^{-1}$ for the radiative and $10^{5} \mathrm{~s}^{-1}$ for the three-body. In principle both mechanisms could be responsible for the $\bar{H}$ production in ATHENA. Important insights into the formation mechanism and state distribution can therefore be obtained by studying the temperature and density dependence of the production of antihydrogen. In a previous publication we reported the temperature dependence [10], in which we measured, for the first time, $\bar{H}$ production as a function of the positron plasma temperature from $15 \mathrm{~K}$ up to more than $3000 \mathrm{~K}$. A clear decrease of the antihydrogen production with the positron plasma temperature has been seen, but a simple power law scaling does not fit the data (see Fig. 4). The naive three-body temperature dependence $\left(T^{-9 / 2}\right)$ is not consistent with our data and the expected predominance of this mechanism below $\sim 100 \mathrm{~K}$ is not supported by the leveling-off at low temperatures. It is important to note that this is the dependence for the $\bar{H}$ atoms that survived trap electrodes and $e^{+}$plasma fields and annihilated to the trap walls. The fall-off in antihydrogen production is slow enough that it is still measurable at room temperature in the ATHENA apparatus.

Important results of the ATHENA experiment were also the study of the dynamics of the antiproton cooling in a positron plasma during antihydrogen formation [9] and the three-dimensional imaging of antiprotons in a Penning trap[19]. In the first case the time evolution of the cooling process has been studied in detail and several distinct phenomena identified. In the second case, by reconstructing the annihilation vertices, the spatial distribution of the antiproton loss, in absence of positrons, was observed. The 
radial loss of $\bar{p} \mathrm{~s}$ on the trap wall was localized to small spots, strongly breaking the azimuthal symmetry expected for an ideal trap.
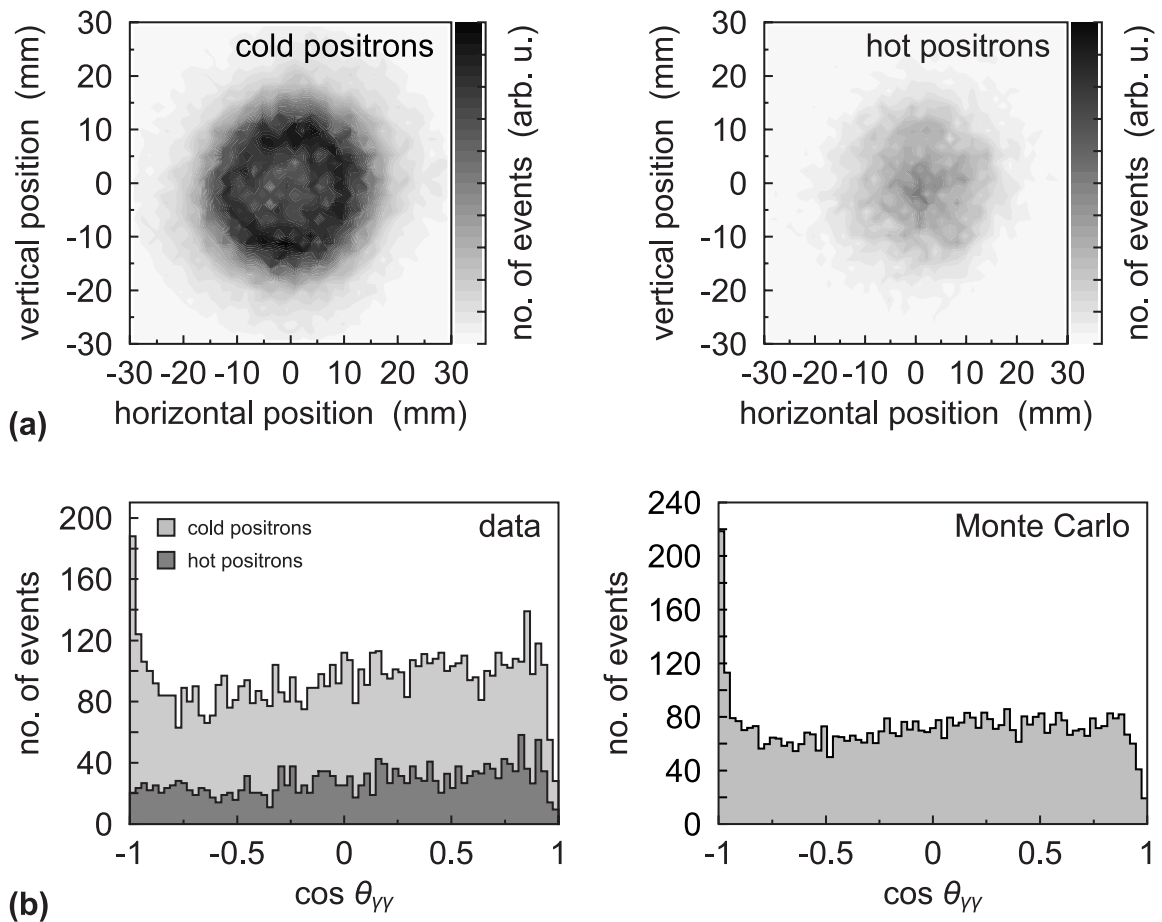

Figure 3: Signal of the first production of cold antihydrogen by ATHENA [3]. (a) Chargedpion vertex distribution as a function of the azimuthal coordinates. (b) Openingangle distribution of the photons recorded in coincidence with the chargedparticle hits, as seen from the charged vertex.

Last in order of time was the study of the spatial distribution of cold antihydrogen formation [21]. Using the antihydrogen annihilation detector, experimental evidence that spatial distribution of the emerging antihydrogen atoms is independent of the positron temperature and axially enhanced was obtained. This indicates that antihydrogen is formed before the antiprotons are in thermal equilibrium with the positron plasma. Using a model in which $\bar{p}$ s rotate with the $e^{+} \mathrm{s}$, and homogenous formation assumed, a lower limit of $150 \mathrm{~K}$ for the axial temperature of the antiprotons was obtained.

\subsection{Laser stimulated recombination}

During 2004 data taking the experimental apparatus was modified to allow the insertion of laser light into the mixing trap to attempt the first laser stimulation of antihydrogen 
production. In particular a $\mathrm{CO}_{2}$ continuous wave laser was used with a tunable wavelength range from9.5 $<\lambda<11.2 \mu \mathrm{m}$. To stimulate radiative $\bar{H}$ production from the continuum to the $n=11$ quantum states, $\lambda=10.96 \mu \mathrm{m}$ was used. The beam waist in the mixing region was about $2 \mathrm{~mm}$ with a typical peak intensity of $160 \mathrm{~W} \mathrm{~cm}^{-2}$ at $10 \mathrm{~W}$ power. The anticipated stimulated formation rate was $60 \mathrm{~Hz}$ under equilibrium conditions at $15 \mathrm{~K}$ and this was not expected to be affected by the finite Doppler width for $\mathrm{T}=15 \mathrm{~K}$ nor by the laser band width (100 MHz). To assure the same conditions for cold mixing with laser light on and off, the comparison has been made in the same mixing cycle, by chopping the beam at a frequency of $25 \mathrm{~Hz}$, with triggers recorded by the DAQ. With the laser on a slight increase in temperature and no vacuum deterioration have been measured. The analysis of the data collected is still in progress.
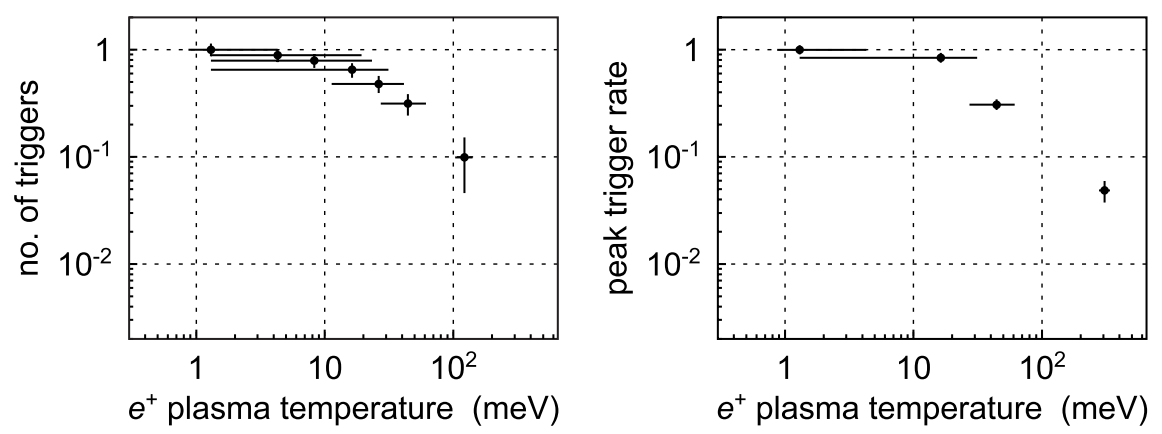

Figure 4: Dependence of the background-corrected integrated total number of chargedparticle triggers per mixing cycle (left) and the peak trigger rate (right) on the positron plasma temperature [10]. The number of triggers and trigger rate have been normalized to the signal for an $e^{+}$temperature of $1.3 \mathrm{meV}(15 \mathrm{~K})$. Note the logarithmic scale.

\section{Conclusions}

The ATHENA experiment produced and detected for the first time cold antihydrogen atoms. Between 2002 and 2004 more than 2 millions anti-atoms were produced. The conditions to routinely produce anti-atoms at an average rate of $10-30 \mathrm{~Hz}$ for a minute are well established. The dependence of the antihydrogen formation on the temperature of the positron plasma, the spatial distribution of the antihydrogen atoms, the cooling process of antiprotons inside a very dense positron plasma, the annihilation patterns of antiprotons (in absence of positrons) inside a Penning trap have been studied for the first time. Some of the challenges on the way to high-precision CPT tests with antimatter have been surmounted, but many still remain. Indeed recombination possibly sets in before complete thermalization causing $\bar{H}$ to be produced with an axial velocity that could make confinement inside a magnetic trap difficult. And confinement is an absolute requirement for high-precision spectroscopic and interferometric measurements with $\bar{H}$. 


\section{References}

[1] G. Baur et al., Phys. Lett. B 368 (1996) 251.

[2] G. Blanford et al., Phys. Rev. Lett. 80 (1998) 3037.

[3] M. Amoretti et al., Nature 419 (2002) 456.

[4] G. Gabrielse et al., Phys. Rev. Lett. 89 (2002) 213401.

[5] G. Gabrielse et al., Phys. Lett. A 129 (1988) 38.

[6] M. Amoretti et al., Nucl. Instr. and Meth. A 518 (2004) 679.

[7] F. Y. Hémery and S. Maury, Nucl. Phys. A 655 (1999) 344c.

[8] T. M. O’Neil, Phys. Fluids 23 (1980) 725.

[9] M. Amoretti et al., Phys. Lett. B 590 (2004) 133.

[10] M. Amoretti et al., Phys. Lett. B 583 (2004) 59.

[11] C. Regenfus, Nucl. Instr. and Meth. A 501 (2003) 65.

[12] M. Amoretti et al., Phys. Rev. Lett. 91 (2003) 055001.

[13] M. Amoretti et al., Phys. Plasmas 10 (2003) 3056.

[14] M. Amoretti et al., Phys. Lett. B 578 (2004) 23.

[15] M.H. Holzscheiter, M. Charlton and M.M. Nicto, Phys. Rep. 402 (2004) 1.

[16] A. Müller and A. Wolf, Hyp. Int. 109 (1997) 233.

[17] J. Stevefelt, J. Boulmer and J-F. Delpech, Phys. Rev. A 12 (1975) 1246.

[18] M. E. Glinsky and T. M. O'Neil, Phys. Fluids B 3 (1991) 1279.

[19] M. Fujiwara et al., Phys. Rev. Lett. 92 (2004) 065005.

[20] G. Gabrielse et al., Phys. Rev. Lett. 89 (2002) 233401.

[21] N. Madsen et al., Phys. Rev. Lett. 94 (2005) 033403. 